\title{
Perceived direction of rotation of simulated three-dimensional patterns
}

\author{
MYRON L, BRAUNSTEIN \\ University of California, Irvine, California 92717
}

\begin{abstract}
Direction of rotation judgments were obtained from 72 subjects for computer-generated dot patterns simulating points randomly distributed in a sphere rotating about a vertical axis. The displays were produced either with normal polar projections or with perspective effects limited to the horizontal or to the vertical dimension of the projection. The simulated viewing distance used in the projections and the visual angle subtended by the projected displays were also varied. Accuracy of direction judgments was about the same with perspective effects limited to the vertical dimension as with normal polar projections but did not exceed chance expectations with perspective effects limited to the horizontal dimension. Accuracy was lower at the greater simulated viewing distance and at the greater visual angle.
\end{abstract}

A primary concern in the study of the perception of motion in depth has been the identification of the processes by which observers judge whether portions of a visual display are approaching or receding. Most of the research on this problem has concentrated on direction of rotation judgments for outline figures rotating about a vertical axis perpendicular to the line of sight (Braunstein, 1976, chap. 6). Braunstein and Payne (1968), using rectangular and trapezoidal grid patterns with height-to-width ratios of approximately $4: 3$, rotating about a vertical axis, found perspective changes in the dimension of the axis of rotation to be the primary source of information used in direction of rotation judgments, with changes in the perpendicular dimension of the projection providing a secondary source. (When the axis of rotation is vertical, as has been the case in almost all studies of perceived direction of rotary motion, these changes are conveniently referred to as vertical and horizontal perspective, respectively.)

Hershberger, Stewart, and Laughlin (1976) compared the effects of horizontal and vertical perspective changes when the two dimensions of change provided conflicting indications of direction of rotation. Although the stimulus display used was one in which horizontal changes were more extreme than vertical changes (because of a width-to-height ratio of 20:1 in the initial dimensions of the pattern) and horizontal changes were more salient (there were 12 equal horizontal gradations and no vertical gradations), direction judgments were determined by vertical perspective effects during most (about 60\%)

This research was supported by National Science Foundation Grant GB-40207. The author is grateful to Wade Arens for his assistance in the conduct of the experiments and to John W. Payne for his comments on an earlier draft of this paper. Requests for reprints should be send to Myron L. Braunstein, School of Social Sciences, University of California, Irvine, California 92717. of the observation periods, as long as the same viewing distance was simulated in the computation of both dimensions of the projected display.

The present study considers the applicability of the results with two-dimensional patterns to the more general case of three-dimensional patterns rotating in three-dimensional space. The pattern selected for investigation consists of small dots randomly dispersed throughout an unseen sphere. There were several reasons for this choice: This pattern contains no planar subpatterns as would be present, for example, in a cube. The presence of such subpatterns would make it difficult to distinguish between the effects of changes in the projection of the individual planes and the effects of projected motions in depth that can occur even in the absence of twodimensional shapes. Second, the width-to-height ratio of $1: 1$ assures that the relative effects of horizontal and vertical perspective changes are not due merely to inequalities in the pattern's dimensions. Finally, the random spacing of the dots avoids the presence of linear perspective effects in momentary views of the rotating pattern.

In the present study, as in Braunstein and Payne (1968), the effects of vertical and horizontal perspective changes were separated by including projections in which polar perspective effects were confined to either the horizontal or to the vertical dimension of the projection, with the other dimension determined by a parallel projection. Parallel projections provide no information from which direction of rotation can be determined, so that any direction information would have to be derived from the dimension in which polar perspective effects were displayed.

Accuracy of direction of rotation judgments for patterns of dots randomly distributed throughout an unseen sphere was previously studied by 
Braunstein (1966a). The patterns were rotated about either a horizontal or a vertical axis, and displayed by means of either a parallel or a polar projection. Accuracy did not exceed chance expectations for the parallel projections, but averaged $86 \%$ for the polar projections. There was little effect of axis of rotation. A vertical axis was selected for the present study, for comparability to most other research on direction of rotation.

Polar projections of dot patterns rotating about a vertical axis display vertical and horizontal perspective effects both in the projected paths and velocities of individual dots and in the relationships among dots. Vertical perspective effects for an individual dot are shown in Figure 1a. The projection of the half of a dot's circular path closest to the projection point (the simulated position of the observer's eye) differs from the projection of the more distant half of that path in both direction and degree of curvature.

The projections of two dots in the same X-Y plane that are vertically aligned will increase in vertical separation as the dots approach the observer and decrease in vertical separation as they recede. This is analogous to changes in the projected size of a vertical contour of a plane figure that occur as the contour approaches or recedes from the observer. The projections of two dots in the same X-Y plane that are horizontally aligned will change in relative distance from the horizontal axis as the dots approach or recede from the observer. This is analogous to changes in the projected angles between horizontal and vertical contours that occur during the rotation of plane figures. These conditions (common plane and vertical or horizontal alignment) may not be strictly met in random dot spheres, but observers may still be able to make approximate associations between changes in the projected separations of clusters of dots and the approach or recession of these dots.
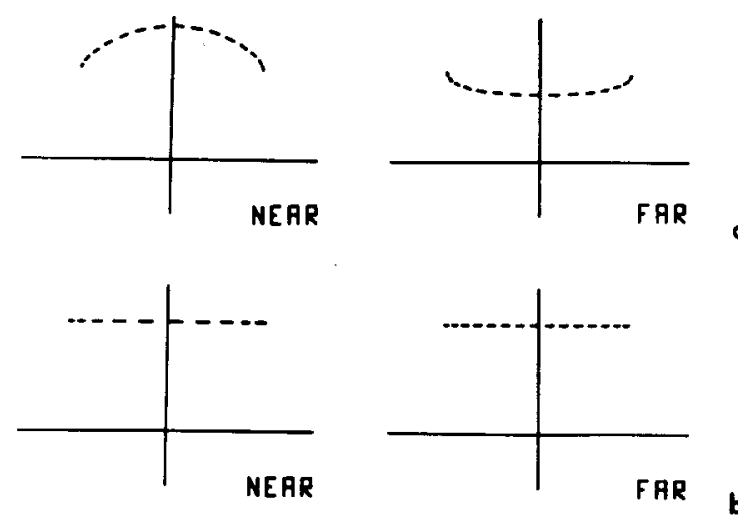

Figure 1. Effects of (a) vertical and (b) horizontal perspective on the projected path and velocity of a dot rotating about a vertical axis. (Dash length indicates distance covered per unit of time.)
The effects of horizontal perspective for an individual dot rotating about a vertical axis are shown in Figure 1b. The projected path of the dot is a straight line when vertical perspective effects are eliminated. The projected velocity is greater when the dot is in the closer portion of its circular path in three-dimensional space. The projected acceleration of the dot, relative to its displacement from the axis of rotation, is also greater when it is in the closer portion of its three-dimensional path (Hershberger, Carpenter, Starzec, \& Laughlin, 1974; Hershberger \& Urban, 1970).

Horizontal perspective affects the relationships among dots in at least two ways (Hershberger \& Urban, 1970): First, the projections of two dots at opposite ends of a horizontal line will move in the same direction horizontally as the line leaves the frontal plane. When this occurs, the dot moving away from the axis of rotation is approaching in depth. Second, the projections of dots on a line reach their maximum horizontal extents in different orders, depending on direction of rotation. Both of these effects are potentially available as indicators of direction of rotation in three-dimensional random dot patterns as well as in horizontal line patterns, but the detection of relationships of this type may be more difficult in three-dimensional patterns.

The magnitudes of both the vertical and horizontal perspective effects vary inversely with the ratio of the simulated viewing distance to the radius of the sphere. Ratios of 3:1 and 12:1 were included in the present study. If the distance at which the subjects viewed each projection were to correspond to the viewing distance simulated in that projection, the visual angles subtended by the displays in the two distance conditions would vary in a ratio of approximately $4: 1$. To separate the effects of perspective variations within the projection from any possible effects of the overall visual angle subtended by the display, displays produced at each of the two simulated viewing distances were presented at both the visual angle appropriate to that simulated distance and the visual angle appropriate to the other simulated distance.

When the visual angle is inappropriate to the simulated viewing distance, the retinal projection produced by the display will not correspond to that produced by a rigid rotation in direct vision. The possibility that an inappropriate viewing distance leads to a perceived lack of rigidity in the rotating pattern was also studied by asking subjects to rate the rigidity or coherence of the displays, after they had completed their direction of rotation judgments.

\section{EXPERIMENT 1}

\section{Method}

Subjects. The subjects were 24 introductory psychology students whose participation partially fulfilled a course requirement. 
Vision of at least $20 / 40$ (Snellen eye chart) was required in the preferred eye. Half of the subjects were assigned to each of the two levels of visual angle.

Stimuli. The stimuli were computer-generated 180 -frame $16-\mathrm{mm}$ motion picture sequences. Each frame contained approximately 1,000 white dots against a dark background. The sequences represented points randomly distributed within the confines of a unit-radius sphere rotating $360^{\circ}$ about a vertical axis through its center. A uniform distribution of dots was obtained by randomly selecting an $\mathrm{X}, \mathrm{Y}$, and $\mathrm{Z}$ coordinate from a rectangular distribution over the range -1 to +1 and rejecting those points for which $\mathrm{X}^{2}+\mathrm{Y}^{2}+\mathrm{Z}^{2}>1$. A projection point distance of either 3 units or 12 units from the center of the spheres was used in producing two-dimensional projections of the dot pattern. Three types of projections were produced at each projection point distance: a conventional polar projection, a projection in which only vertical perspective changes were displayed, and a projection in which only horizontal perspective changes were displayed. Each of the six combinations of projection distance with projection type were produced with two random dot patterns. The stimulus film consisted of two random arrangements of these 12 sequences, with the first 14 of the sequences in the film repeated at the end. This gave the film 12 possible starting points from which 27 sequences could be presented, with the last three sequences the same as the first three. Twelve additional orders of presentation were obtainable by running the film in reverse.

Apparatus. A 16-mm motion-picture projector (L-W Model 224-A), operated at $24 \mathrm{frames} / \mathrm{sec}$, was used to display the stimulus film. Two of the shutter blades were covered to prevent the perception of multiple images (Braunstein, 1966b). The film was projected onto a translucent screen (Polacoat) in an adjacent room. The subject viewed the screen monocularly from the opposite side, at a distance of $1.8 \mathrm{~m}$, through an arrangement of tubes which restricted the field of view to a circular area of either .3 or $1.2 \mathrm{~m}\left(9.6^{\circ}\right.$ or $\left.39.7^{\circ}\right)$, depending on which of two interchangeable tube systems was used. The diameter of the sphere on the projection screen was matched to the viewing area by changing the projector lens from 100 -mm lens in the small visual angle condition to a $25-\mathrm{mm}$ lens in the large visual angle condition. The viewing area was always filled with dots, i.e., the edge of the sphere was not visible.

The response device was a wooden sphere approximately $5 \mathrm{~cm}$ in diameter, located on a table in front of the subject. The wooden sphere was mounted on a shaft which extended below the table, where it would close one of two microswitches, depending on whether it was turned clockwise or counterclockwise. The microswitches controlled lights at the experimenter's console. A spring mechanism returned the response sphere to its original position when it was released. A 15 -cm-diam glass sphere on which small white dots were unsystematically pasted was used as a demonstration device. It was attached to the shaft of a reversible motor controlled by the experimenter.

Procedure. The subject was instructed to view the displays through the tube with the same eye as that used in the eye-chart test, and to indicate the direction of rotation for each display "immediately after the display goes off." Clockwise and counterclockwise rotations were demonstrated with the glass sphere, and the subject practiced the response procedure. The subject's room was then made totally dark and the film was started. A different order of presentation was obtained for each of the 24 subjects by starting the film at one of the first 12 sequences and running it forward or starting at one of the last 12 sequences and running it in reverse. A total of 27 sequences were presented, with the last three sequences the same as the first three, which were treated as practice trials. The subject was then instructed that, for the second part of the experiment, the displays were to be rated "on apparent coherence or rigidity," defined as "the degree to which the dots seem to maintain the same relative positions as the display moves." A chart was shown to the subject on which the numbers 1-4 were equally spaced along a horizontal line, with the expressions "completely rigid and coherent," "some stretching or elasticity," "a few dots moving independently," and "breaks up into groups of dots," printed above the four numbers, respectively. The sequences were then presented in the reverse of the order used in the first part of the experiment. The responses were made verbally and recorded on tape.

\section{Results}

Direction judgments. The mean proportions of correct responses for each of the six display conditions and two viewing conditions are shown in Table 1. The proportions for the individual subjects, each based on four responses, were subjected to an analysis of variance using an arcsine transformation. The effects of perspective level, $F(1,22)=28.1$, and of perspective type, $F(1,44)=14.0$, and the interaction of perspective level and type, $F(2,44)=16.0$, were significant, $p<.05$. The effect of viewing angle, $F(1,22)=3.26$, and the interactions involving the viewing angle were not significant. The effects of perspective level and type were similar to those found with rectangles and trapezoids (Braunstein \& Payne, 1968): Accuracy was about the same for normal and vertical perspective, but was lower for horizontal perspective. In the present experiment, horizontal perspective accuracy did not exceed chance expectations (binomial test).

Coherence. Mean coherence ratings for the 12 combinations of display type and viewing condition ranged from 2.27 to 2.96 . An analysis of variance showed no significant effects.

\section{EXPERIMENT 2}

Experiment 2 is a replication of the direction judgment portion of Experiment 1, with the lower of the two perspective levels (the 12-unit viewing distance) eliminated. This was for the purpose of obtaining more precise quantitative estimates of the effects of type of perspective, viewing condition, and the interaction of these two variables. There was some indication in Experiment 1 and in pilot studies that the accuracy of direction judgments for higher perspective level displays may be reduced by the presentation of lower perspective level displays on previous trials. This is because subjects tended to repeat the same direction judgments at the lower perspective level,

Table 1

Proportions of Correct Responses in Experiment 1

\begin{tabular}{lccccc} 
& \multicolumn{3}{c}{ Projection Distance } \\
\cline { 2 - 3 } & \multicolumn{2}{c}{ 3-Unit } & & \multicolumn{2}{c}{ 12-Unit } \\
\cline { 2 - 3 } \cline { 5 - 6 } Perspective & \multicolumn{2}{c}{ Visual Angle } & & \multicolumn{2}{c}{ Visual Angle } \\
$\quad$ Type & $9.6^{\circ}$ & $39.7^{\circ}$ & & $9.6^{\circ}$ & $39.7^{\circ}$ \\
\hline Normal & .85 & .75 & & .54 & .50 \\
Horizontal & .54 & .48 & & .58 & .58 \\
Vertical & .90 & .79 & .64 & .50 \\
\hline
\end{tabular}


regardless of the actual direction displayed, and this "set" effect could have affected the direction judgments even for some of the higher perspective level displays. Ideally, the possibility of a set effect could be eliminated by having each subject respond to only one display, but this is not a practical way to compare display variables. Instead, the possible effect of set was diminished by eliminating the lower perspective level stimuli, thereby raising the overall level of accuracy in the experiment.

\section{Method}

Subjects. The subjects were 48 students from the same source as in Experiment 1. The vision criterion was the same as in Experiment 1. Half of the subjects were assigned to each of two levels of visual angle.

Stimuli. The stimulus displays consisted of the six sequences used in Experiment 1 for which the projection distance was three units. A film was prepared containing a random order of the six sequences.

Apparatus. The apparatus was the same as in Experiment 1.

Procedure. The procedure was essentially the same as in Experiment 1, except that no coherence judgments were required. Twelve different orders of presentation were obtained by starting the film at one of the six sequences and running the projector in either the forward or reverse direction. When either end of the film was reached, the direction of film transport was reversed, until a total of 15 sequences were presented. The first three sequences for each subject were the same as the last three, and were treated as practice trials. Four subjects were run in each of the 12 orders of presentation.

\section{Results}

Table 2 shows the mean proportions of correct rotation direction judgments for the three types of perspective and two visual angle conditions. An analysis of variance, using an arcsine transformation of the proportions for the individual subjects, showed significant $(p<.05)$ main effects for the perspective type, $F(2,92)=62.2$, and for visual angle, $F(1,46)=5.36$. The interaction, $F(2,92)=1.82$, was not significant. As in Experiment 1, accuracy was about the same for normal and vertical perspective, but did not exceed chance expectations for horizontal perspective. Accuracy was higher for the smaller visual angle displays. (This trend was also apparent in Experiment 1, but did not reach significance.) A comparison of the results of Experiment 2 with the same conditions in Experiment 1 showed slightly higher accuracy in Experiment 2 for five of the six comparable conditions and the same accuracy for the remaining condition. There was, at most, a small reduction in accuracy in Experiment 1 at the higher perspective level due to the inclusion of the lower perspective displays.

\section{DISCUSSION}

The results of the present investigation are consistent with Braunstein and Payne's (1968) findings with rectangular and trapezoidal grid patterns:
Table 2

Proportions of Correct Responses in Experiment 2

\begin{tabular}{lcc}
\hline & \multicolumn{2}{c}{ Visual Angle } \\
\cline { 2 - 3 } $\begin{array}{c}\text { Perspective } \\
\text { Type }\end{array}$ & $9.6^{\circ}$ & $39.7^{\circ}$ \\
\hline Normal & .90 & .75 \\
Horizontal & .55 & .52 \\
Vertical & .91 & .80 \\
\hline
\end{tabular}

Vertical perspective alone is about as effective as combined horizontal and vertical perspective in determining direction of rotation judgments, for patterns rotating about a vertical axis, but horizontal perspective alone is much less effective. In the present study, horizontal perspective effects never exceeded chance expectations. These results would not be consistent with an explanation of perceived direction of rotation in terms of differences in projected velocity between the front and rear surfaces of the sphere under various perspective conditions, as these velocity differences are determined almost entirely by horizontal perspective changes.

The present results are not inconsistent with those of Hershberger et al. (1976) if the difference in stimulus patterns is taken into consideration. Hershberger et al. obtained proportions of correct responses of approximately .68 and .81 , respectively, with vertical perspective alone, for the two projection distances included in the present experiment, 12 units and 3 units. The proportions in Experiment 1 of the present study were .65 and .90 . For horizontal perspective alone, Hershberger et al. obtained proportions of .66 and .77 at those two distances, considerably higher than the present .58 and .54 . The low accuracy found with horizontal perspective alone in the present study may have been due to difficulty in detecting certain horizontal perspective effects in three-dimensional random dot patterns. Effects based on relationships among the motions of two or more dots, such as periods when the projections of certain dots move in the same direction and the order in which dots reach their maximum distance from the axis of rotation, may be less detectable in threedimensional random dot patterns than in twodimensional patterns showing equal horizontal gradations.

If horizontal perspective effects are less detectable in three-dimensional patterns than in two-dimensional patterns, Hershberger et al.'s (1976) quantitative results with horizontal perspective alone should be more comparable to those obtained by Braunstein and Payne (1968) than to the present results. This indeed appears to be the case. For 3-, 6-, 12-, and 24unit projection distances, Hershberger et al. obtained proportions of correct response time of .77, $.73, .66$, and .56 for horizontal perspective displays. Braunstein and Payne, for 3-, 3.67-, 5-, and 9-unit distances, obtained proportions of approximately 
$.71, .73, .66$, and .56 (estimated by adding the obtained proportion of responses that were correct during a full $360^{\circ}$ rotation to half the proportion of oscillation responses, which averaged .50 correct, in terms of response time.)

Hershberger et al.'s response procedure was also more like that of Braunstein and Payne's procedure than that used in the present experiments. In the Braunstein and Payne and in the Hershberger et al. experiments, subjects were asked to respond continuously during the display presentation; in the present study, a single response was made at the end of each sequence. (The present procedure was adopted because virtually no reversals of apparent direction were found during a sequence in pilot studies with random dot spheres.) It seems unlikely, however, that the reduced accuracy found in the horizontal perspective condition of the present study would have occurred only in that condition if it was the result of a difference in procedure.

Direction judgments were more accurate in the smaller visual angle viewing condition, in which the viewing distance was 12 times the width of the projected sphere, for both displays simulating a 3-unit projection distance and those simulating a 12-unit distance. Accuracy was greatest when the simulated viewing distance was 3 units and the visual angle was based on a viewing distance of 12 units. This was due to the separate effects of the two variables, rather than to an interaction. As the effect of visual angle was unrelated to its appropriateness to the perspective displayed, it would be difficult to reach even a tentative conclusion about this variable without further study. Possibly the smaller angle made it easier to compare changes occurring at opposite edges of the display or to follow the motion of a single dot. There was no evidence that displays appeared more coherent when the simulated viewing distance and visual angle were matched, but the high variability in the coherence ratings may have obscured any such effect.

Although random dot spheres, like virtually all stimulus materials used in laboratory studies of visual perception, are just abstractions from the types of stimulation encountered in normal vision, they are probably more characteristic of this stimulation than are the plane figures usually studied. Random dot spheres have the internal depth and texture irregularity typical of natural visual scenes.
For this reason, it is important that earlier findings concerning the relative effectiveness of vertical and horizontal perspective generalize to these stimuli. The next question to be asked about direction of rotation judgments for three-dimensional patterns concerns the specific vertical perspective changes that account for direction judgments, a question paralleling that addressed by Börjesson (1971) and Braunstein (1971) for selected two-dimensional figures and by Hershberger (for example, Hershberger et al., 1974; Hershberger \& Urban, 1970) for horizontal perspective changes in rows of dots. Another question would concern the circumstances under which horizontal perspective changes might become more effective for three-dimensional patterns. Increasing the width-to-height ratio of the pattern might have this effect, as might adding regularity to the horizontal spacing, but caution would have to be exercised in generalizing from the stimuli resulting from such manipulations to those encountered in direct vision.

\section{REFERENCES}

BöRJEsson, E. Properties of changing patterns evoking visually perceived oscillation. Perception \& Psychophysics, 1971, 9, 303-308.

Braunstein, M. L. Sensitivity of the observer to transformations of the visual field. Journal of Experimental Psychology, 1966, 72, 683-689. (a)

Braunstein, M. L. Interaction of flicker and apparent movement. Journal of the Optical Society of America, 1966, 56, 835-836. (b)

Braunstein, M. L. Perception of rotation in figures with rectangular and trapezoidal features. Journal of Experimental Psychology, 1971, 91, 25-29.

Braunstein, M. L. Depth perception through motion. New York: Academic Press, 1976.

Braunstein, M. L., \& Payne, J. W. Perspective and the rotating trapezoid. Journal of the Optical Society of A merica, 1968, 58, 399-403.

Hershberger, W. A., Carpenter, D. L., Starzec, J., \& LAUGHLIN, N. K. Simulation of an object rotating in depth: Constant and reversed projection ratios. Joumal of Experimental Psychology, 1974, 103, 844-853.

Hershberger, W. A., Stewart, M. R., \& Laughlin, N. K. Conflicting motion perspective simulating simultaneous clockwise and counterclockwise rotation in depth. Journal of Experimental Psychology: Human Perception and Performance, 1976, 2, 174-178.

HeRshbERGER, W. A., \& URBAN, D. Three motion-parallax cues in one-dimensional polar projections of rotation in depth. Journal of Experimental Psychology, 1970, 86, 380-383.

(Received for publication November 29, 1976; revision accepted March 14, 1977.) 